\title{
PHYSIOLOGICAL EVIDENCE FOR SPECIFICITY OF SYNAPTIC CONNECTIONS BETWEEN INDIVIDUAL SENSORY AND MOTOR NEURONS IN THE BRACHIAL SPINAL CORD OF THE BULLFROG ${ }^{1}$
}

\author{
JEFF W. LICHTMAN ${ }^{2}$ AND ERIC FRANK ${ }^{3}$ \\ Department of Neurobiology, Harvard Medical School, Boston, Massachusetts 02115 \\ Received October 10, 1983; Revised January 26, 1984, Accepted January 30, 1984
}

\begin{abstract}
We have studied synaptic connections between individual stretch-sensitive muscle afferents and motoneurons in the brachial spinal cord of bullfrogs. Sensory afferents from a given head of the triceps brachii muscle preferentially innervate motoneurons that project to the same muscle head. This preference is characterized in two ways: each class of sensory axon innervates a greater proportion of corresponding motoneurons than of motoneurons projecting to synergistic or unrelated muscles, and the synaptic potentials in these corresponding motoneurons are of larger amplitude. A novel feature of these experiments is that even the smallest averaged synaptic potential is several times larger than the noise level. These smallest synaptic potentials thus represent the smallest synaptic interaction between a sensory and motor cell, and they could be the physiological correlate of a single sensory bouton on a motoneuronal dendrite.
\end{abstract}

How specific connections are made between nerve cells and their targets is a fundamental but as yet poorly understood aspect of the development of the nervous system. Although there have been many suggestions regarding how the specificity of neural connections arises, it has been difficult to describe synaptic patterns at a sufficiently detailed level to assess these ideas critically. Moreover, many of the more detailed descriptions of neural specificity have been made in relatively simple parts of the nervous system such as the neuromuscular junction or peripheral autonomic ganglia, where the basis of specificity might be different from that in the central nervous system. In this study, we have attempted to describe in detail the specificity within a set of synaptic connections in the central nervous system where hundreds or thousands of inputs converge on a single postsynaptic neuron.

\footnotetext{
${ }^{1}$ It is a pleasure to acknowledge useful suggestions and criticisms from S. Jhaveri, P. C. Jackson, and D. Sah, and expert technical assistance from J. Gagliardi, M. Nastuk, Y. Pearlson, M. Peloquin, and E. Wu. This work was supported by National Institutes of Health Grant NS 14451 and National Science Foundation Grant BNS-8021605 to E. F.

${ }^{2}$ To whom correspondence should be sent, at his present address: Department of Physiology and Biophysics, Washington University School of Medicine, 660 South Euclid Avenue, St. Louis, MO 63110.

${ }^{3}$ Present address: Department of Neurobiology and Physiology, Northwestern University, Evanston, IL 60201.
}

The system we have studied is the myotatic or stretch reflex, a monosynaptic reflex loop between sensory afferents and motor neurons in the spinal cord. Many stretch-sensitive afferent axons converge on each motoneuron, yet the entire input from all such afferents forms only a small fraction of the total input. The long history of study of this reflex provides a wealth of information concerning the specificity of these connections in mammals (Lloyd, 1943, 1946; Eccles et al., 1957). In fact, it is one of the few systems in the central nervous system where it has been possible to study the physiological projection patterns of individual axons (see, for example, Kuno, 1964; Mendell and Henneman, 1971; Scott and Mendell, 1976; Watt et al., 1976).

The bullfrog provides a useful preparation to study these connections. The spinal cord, brachial nerve, and arm muscles can be entirely dissected in continuity and free from the rest of the animal. Previous studies demonstrated that electrical stimulation of the sensory axons in the triceps muscle nerve evokes larger excitatory synaptic potentials in triceps motoneurons than in motoneurons innervating other arm muscles (Frank and Westerfield, 1982). In the present study we activated the sensory axons physiologically by stretching the muscle. By using a technique that allowed us to activate individual stretch-sensitive axons while recording from identified motoneurons, we were able to compare the influences of afferent axons from several muscles on one motoneu- 
ronal pool and, conversely, of stretch-sensitive afferents from one muscle on several motor pools.

\section{Materials and Methods}

\section{Dissection}

Young adult bullfrogs (Rana catesbeiana), 5 to $10 \mathrm{~cm}$ in length (Amphibians of North America, Nashville, TN), were anesthetized in ice water and decapitated. The brachial spinal cord was exposed by dorsal laminectomy and the three heads of the triceps brachii muscle were dissected such that the medial head was separated entirely from the combined internal and external heads. All three heads of the triceps muscle were removed from the arm in continuity with the brachial nerve. The spinal cord was then hemisected and completely removed from the vertebral canal, together with the spinal roots and dorsal root ganglia. The preparation thus consisted of an isolated, hemisected brachial spinal cord with intact dorsal and ventral roots in continuity through the brachial nerve with the triceps muscle (see Fig. 1). The preparation was bathed with oxygenated saline at $14^{\circ} \mathrm{C}$ (Frank and Westerfield, 1982).

\section{Electrophysiological recording techniques}

Action potentials in stretch-sensitive axons were recorded en passant in the muscle nerve using a fine platinum wire hook that was used to pull the nerve to one or all three triceps muscles into a capillary tube filled with mineral oil. Recordings were made differentially between the hook and the bath. In separate experiments, used to determine the number of sensory axons in a muscle nerve, we recorded from the peripheral stump of cut dorsal roots using conventional, tight-fitting suction electrodes.

Stretch-sensitive muscle afferents were activated by tapping the muscle surface at sensitive regions with a small glass rod fastened to a piezoelectric transducer. Sensitive regions, presumably near sensory endings, were identified by recording from the muscle nerve (Figs. 1 and 2); each tap (1 msec duration) elicited a single action potential. Axons could follow these taps faithfully up to about $50 \mathrm{~Hz}$, although we used much slower rates (2 to $10 \mathrm{~Hz}$ ) for most experiments. Occasionally two receptors were activated by single taps as revealed by two impulses with nearly identical latencies recorded by the hook electrode. Since the latency of each impulse fluctuated

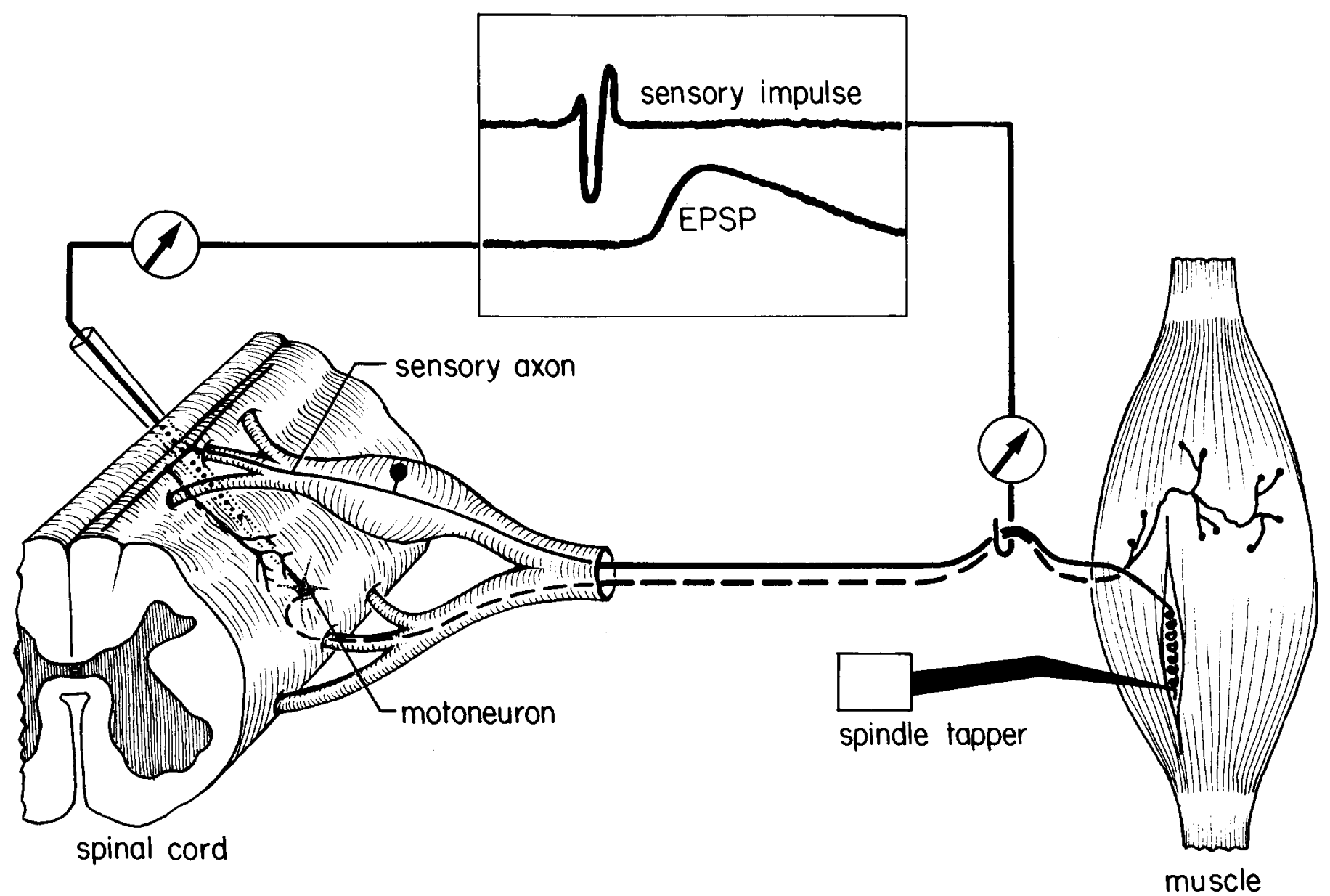

Figure 1. Diagram of the experimental preparation. The hemisected brachial spinal cord with dorsal and ventral roots intact was dissected in continuity with the brachial nerve and all three triceps muscle heads (only one shown here). Tapping a stretchsensitive sensory ending in the muscle elicited an action potential in a sensory axon that could be recorded en passant with a hook electrode placed under the triceps nerve. A simultaneous intracellular recording from a motoneuron innervating the same muscle shows an EPSP following the sensory impulse. Repetitive taps of the same sensory ending were used to measure the average amplitude of the individual EPSPs. See the text for details. 


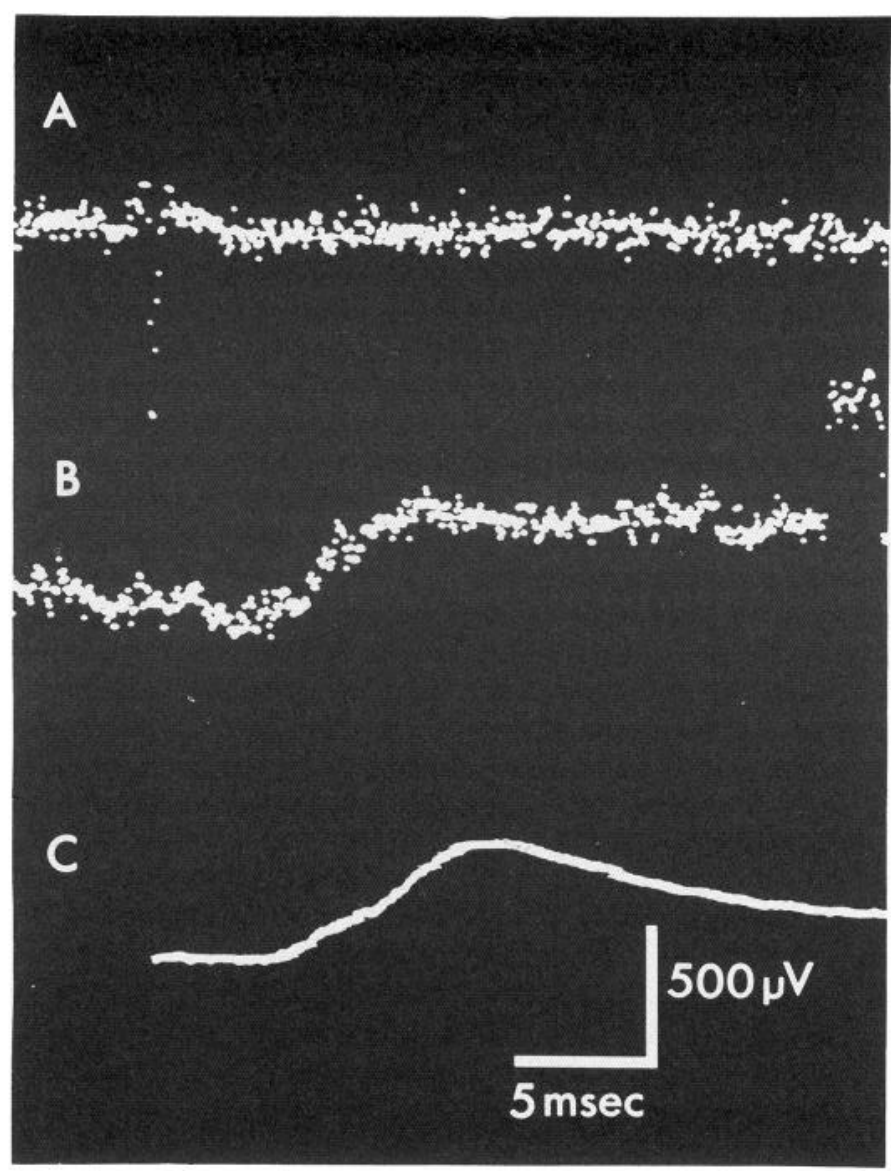

Figure 2. Electrical recordings illustrating the technique of spike-triggered averaging. The sensory impulse shown in trace $A$ was recorded en passant from the triceps muscle nerve (see Fig. 1), and it elicited an EPSP in a homonymous triceps motoneuron (trace $B$ ). These two traces correspond to the two shown schematically in Figure 1. The sensory impulse was used to trigger a signal averager; the resulting average of 256 individual EPSPs is shown in trace $C$. In this example, two components of the EPSP can be distinguished: an early one mediated electrically and a later one mediated chemically (see Shapovalov and Shiriaev, 1980; Westerfield and Frank, 1982). The time calibration applies to all three traces; the voltage calibration applies to traces $B$ and $C$. A calibration pulse $(0.5$ $\mathrm{mV}, 2 \mathrm{msec}$ ) occurs on the falling phase of the EPSP in trace $B$.

slightly from trial to trial, they rarely occurred at exactly the same time; thus, it was obvious that two independent receptors were being activated. In these cases, repositioning the glass probe eliminated one of the responses. Stretch-sensitive axons were also activated by stretching the muscle. A sustained stretch gave rise to a sustained barrage of activity in many, if not all, sensory axons from that muscle.

Motoneurons were impaled with glass microelectrodes filled with $0.5 \mathrm{M}$ potassium citrate (100 to 120 megohms). The electrode was inserted ventrolaterally from the cut surface of the hemisected spinal cord. We accepted only those impalements in which the resting potential was 50 $\mathrm{mV}$ or greater. Impaled cells were identified as motoneurons by antidromic stimulation from the dissected muscle nerves and, in the case of triceps motoneurons, by ob- serving muscle contraction in one or more heads of the triceps muscle during activation of the motoneuron with depolarizing current from the recording microelectrode.

Excitatory postsynaptic potentials (EPSPs) were recorded in motoneurons following activation of triceps sensory axons. Because these potentials were small and mixed with a continuous barrage of spontaneous synaptic potentials unrelated to the stretch reflex, it was necessary to average single evoked events to obtain accurate amplitude measurements. The sensory action potential recorded peripherally was used as a trigger for the oscilloscope and the signal averager, as originally described by Mendell and Henneman (1971). Consecutive traces were averaged until the amplitude could be measured accurately. Examples are shown in Figure 2: the sensory impulse in the upper trace, a single intracellular record in the middle trace, and the average of 256 such single traces in the lower record. In cases where no synaptic potential was seen (a projection "failure"), the averaging procedure was continued until fluctuations in the trace were less than $10 \mu \mathrm{V}$ in amplitude. This procedure made it possible to resolve all synaptic potentials whose average amplitude was $10 \mu \mathrm{V}$ or more. Errors are given as 1 SEM.

Projections of motoneurons. Although we found some motoneurons projecting exclusively to each triceps muscle head, our results indicated that triceps motoneurons could be subdivided into two groups: those that projected exclusively to the medial head and those that projected to one or both of the internal and external heads. About one-third of the motoneurons in the latter group projected to both muscles, and muscle sensory afferents of any one class showed no difference in their innervation of pure internal, pure external, or internal-external motoneurons. The origins and insertions of the internal and external triceps muscles are also very similar, and they have been considered functionally as a single muscle (Frank and Westerfield, 1982; Westerfield and Frank, 1982). Therefore, in the results presented here, we have considered the various types of internal and external triceps motoneurons as belonging to a single class.

Types of motoneurons. In recording from motoneurons that innervate the triceps muscle, we found evidence for three different types. The most prevalent class $(72 \%)$ caused a visible twitch in the triceps muscle each time the motoneuron was brought to threshold. About $24 \%$ of the cells behaved more like slow twitch motoneurons (see, for example, Ridge, 1971), in that a single impulse in these cells usually did not evoke a visible twitch in the muscle; instead a graded contraction developed with repetitive stimulation at 5 to $10 \mathrm{~Hz}$. Both of these cell types had conduction velocities of 10 to $20 \mathrm{~m} / \mathrm{sec}$. This second type of motoneuron, which apparently has not been described before in the frog, may correspond to the motor axons innervating frog muscle fibers with subthreshold synaptic inputs, as described by Herrera and Grinnell (1980). Rarely, we encountered motoneurons of a third type that appeared truly tonic, since they caused a very slow contraction only visible with repetitive stimulation at $20 \mathrm{~Hz}$ or greater. These slow motoneurons had conduction velocities of only 2 to $5 \mathrm{~m} / \mathrm{sec}$, and it was difficult to maintain a recording from them, probably 
due to their smaller size. We found no significant differences in the pattern of sensory innervation to the two twitch type motoneurons. The small sample of slow motoneurons also suggested no major differences. In the results presented here, therefore, we have not distinguished among the three different types.

\section{Histological procedures}

To determine the number of sensory spindles in the triceps muscles, whole muscles were embedded in methacrylate (JB4, Sorvall) and sectioned transversely at 5 $\mu \mathrm{m}$. The sections were stained with Mallory's trichrome and examined in the light microscope. Camera lucida drawings of the sections were used to trace individual intrafusal fiber bundles from one section to the next.

To count sensory axons in the triceps nerves, we destroyed the motor axons by cutting the ventral root 2 weeks before fixation. Segments of the peripheral nerves near the muscles were then embedded in plastic, and 1to $2-\mu \mathrm{m}$ cross-sections were taken every $100 \mu \mathrm{m}$. Profiles of myelinated axons were counted from camera lucida drawings made with an oil immersion objective. Each nerve was examined at 6 to 10 different levels along its length.

\section{Results}

We assessed the pattern of synaptic connections that sensory axons from the three heads of the triceps brachii muscle make with motoneurons projecting to the triceps and other arm muscles. The input from individual axons was measured as was the average input to motoneurons from all stretch-sensitive axons from a particular muscle.

\section{Synaptic potentials and projection frequencies of individual sensory axons}

The central projections of individual stretch-sensitive axons were measured by activating single sensory receptors in a muscle with a small probe while recording intracellularly from an identified motoneuron (see "Materials and Methods" and Figs. 1 and 2). We examined the synaptic projections of a given triceps sensory afferent onto homonymous motoneurons (those innervating the same triceps muscle head), heteronymous motoneurons (those innervating a different head of triceps), and "unrelated" motoneurons (those innervating a separate arm muscle). By moving the probe to different places on the surfaces of the three triceps heads, we could activate several different receptors and thus study the projection of several individual axons onto a single motoneuron. The EPSPs evoked by 12 different triceps sensory axons in a single internal-external triceps motoneuron are shown in Figure 3.

Although there was great diversity in the amplitude of the different individual inputs, homonymous triceps inputs were, on average, larger than heteronymous ones. Sensory inputs to homonymous motoneurons were 195 $\pm 13 \mu \mathrm{V}$ (medial to medial) and $140 \pm 10 \mu \mathrm{V}$ (internalexternal to internal-external), whereas heteronymous inputs were only $78 \pm 9 \mu \mathrm{V}$ (medial to internal-external) and $55 \pm 6 \mu \mathrm{V}$ (internal-external to medial). The difference in amplitude between homonymous and heteronymous connections is due both to a greater projection
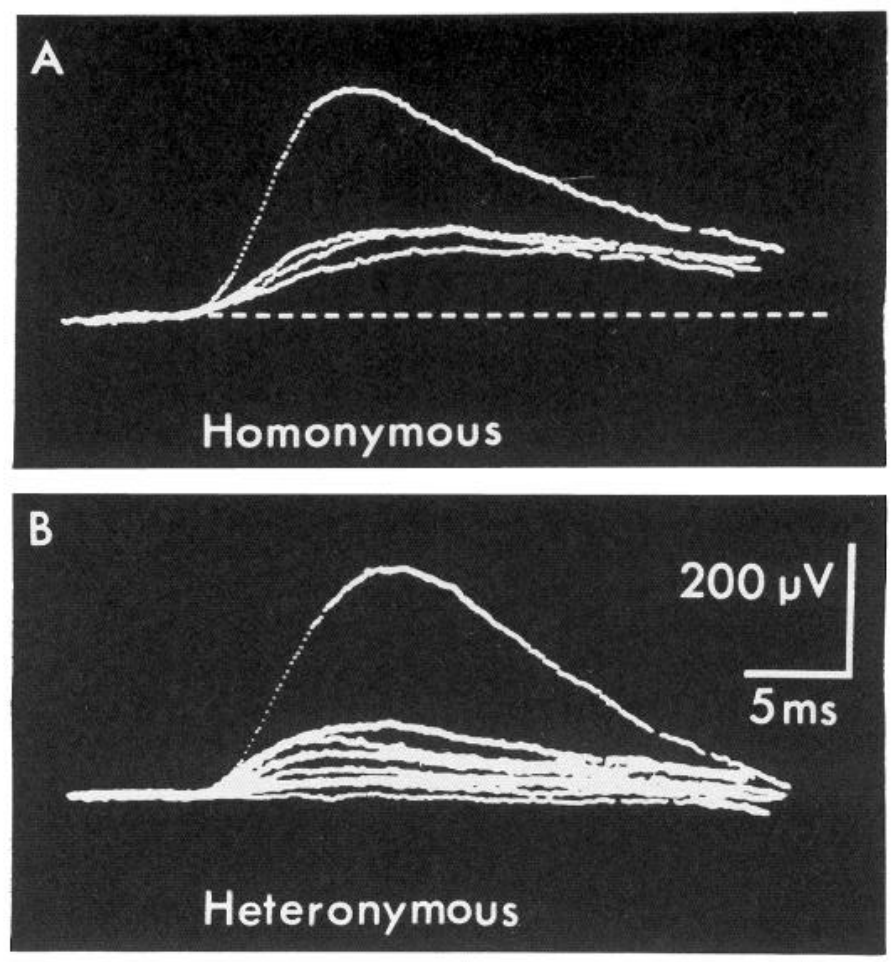

Figure 3. Unitary sensory-motor EPSPs recorded in a single internal-external triceps motoneuron elicited by tapping a number of different locations on the internal and external heads (homonymous) and the medial head (heteronymous). Each trace shows the average EPSP elicited by activation of a single sensory axon; up to several hundred individual traces were averaged to produce each trace shown here. Only four sensitive locations could be found in the combined internal and external heads, compared to more than 10 locations on the medial head. Several sensitive locations that elicited an impulse recorded en passant on the medial muscle elicited no detectable EPSP, but only one example is included in the figure. In $A$, the base line is indicated by a dashed line. The range of homonymous and heteronymous EPSP amplitudes is large, but in general homonymous EPSPs are larger than heteronymous ones.

frequency of sensory axons to homonymous motoneurons and to a greater amplitude of homonymous EPSPs (i.e., excluding the failures of projection). This is seen clearly by comparing the amplitude histograms of the various classes of EPSPs (Fig. 4). Thus, individual medial triceps sensory axons innervated $94 \%$ (106 of 113$)$ of the homonymous motoneurons and only $60 \%$ ( 80 of 133 ) of the heteronymous cells. Similarly, internal-external triceps sensory axons projected to $80 \%$ (138 of 173) of the homonymous cells and only $58 \%$ (74 of 129) of the heteronymous ones. The average homonymous versus heteronymous EPSPs (excluding failures of projection) were $207 \mu \mathrm{V}$ versus $129 \mu \mathrm{V}$ for medial triceps sensory afferents, and $176 \mu \mathrm{V}$ versus $96 \mu \mathrm{V}$ for internal-external triceps afferents.

Triceps sensory axons project only weakly, if at all, to at least two other classes of motoneurons located in the same region of the spinal cord. Subscapularis and pectoralis motoneurons received virtually no innervation from any of the 52 triceps afferents tested (Fig. 4, OTHER). 


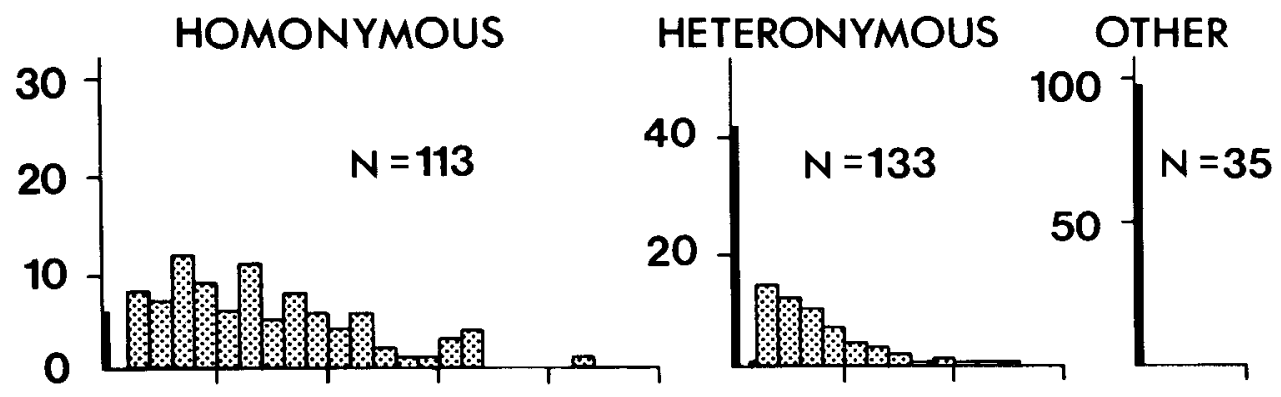

EPSP Amplitude from Individual Medial Triceps Sensory Axons, mV

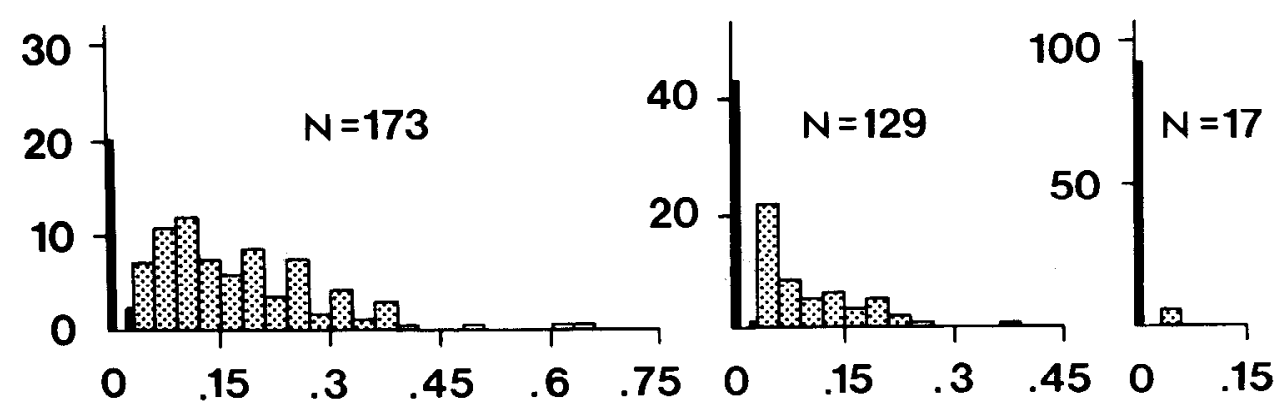

EPSP Amplitude from Individual Internal-External Triceps Sensory Axons, $m V$

Figure 4. Amplitude histograms of averaged EPSPs from individual medial and internal-external triceps sensory axons in three types of motoneurons. The first bin in each histogram has been subdivided into three bins, each $10 \mu \mathrm{V}$ wide, to illustrate that the smallest projections were well above the noise level. The right-hand histograms $(O T H E R)$ represent the combined results from subscapularis and pectoralis motoneurons which are located in the same region of the spinal cord. Homonymous projections are larger and more frequent than heteronymous ones. The two types of unrelated motoneurons receive virtually no input from triceps sensory axons. Note that one large EPSP of $0.70 \mathrm{mV}$ amplitude is not included in the upper middle histogram, and one of $0.78 \mathrm{mV}$ is not included in the lower left histogram.

\section{Minimum amplitude of EPSPs}

An unexpected finding in these experiments was that the smallest averaged synaptic potentials measured were 3 to 4 times larger than the "noise" level. To illustrate this, the first bin of each of the histograms in Figure 4 has been subdivided into three bins (each is $10 \mu \mathrm{V}$ wide). There is a clear separation between "failures" $(<10 \mu \mathrm{V}$, which is the smallest response we could detect) and the smallest measurable synaptic responses (30 to $40 \mu \mathrm{V}$ ). The separation was apparent for all classes of sensorymotor pairs. This suggests that the smallest averaged EPSPs we have measured represent the smallest synaptic connections that exist between sensory and motor neurons in this preparation.

\section{Average projections of sensory axons}

The amplitudes of homonymous and heteronymous triceps EPSPs were compared in another way, by measuring the average unitary input a motoneuron received from many different sensory axons. Each head of the triceps muscle was stretched independently to evoke a continuous barrage of sensory impulses (from all or most of the muscle spindles in that muscle) while we recorded intracellularly from a triceps motoneuron. The impulse frequency was kept low (by applying only weak stretches) to avoid summation of the individual EPSPs. An impulse in any of the stretch-activated sensory fibers triggered the signal averager and thereby added the EPSP it evoked in the motoneuron to the average. The traces shown in Figure 5 show the average of 256 individual EPSPs and represent the average unitary input the motoneuron received from sensory axons in the muscle that was stretched.

We found that, just as for the case of individually activated sensory axons, the average homonymous input was always larger than the heteronymous one. The results of recordings from 25 motoneurons were $181 \pm 25$ $\mu \mathrm{V}$ (medial to medial) and $154 \pm 14 \mu \mathrm{V}$ (internal-external to internal-external) versus $69 \pm 9 \mu \mathrm{V}$ (medial to internal-external) and $57 \pm 5 \mu \mathrm{V}$ (internal-external to medial). The similarity of the results obtained by the two different methods suggests that both probably provide reliable estimates of average homonymous and heteronymous inputs.

To summarize, individual triceps sensory axons project more frequently and establish more effective synapses with motoneurons innervating their own muscle than they do with synergistic motoneurons. Both homonymous and heteronymous connections are in marked con- 

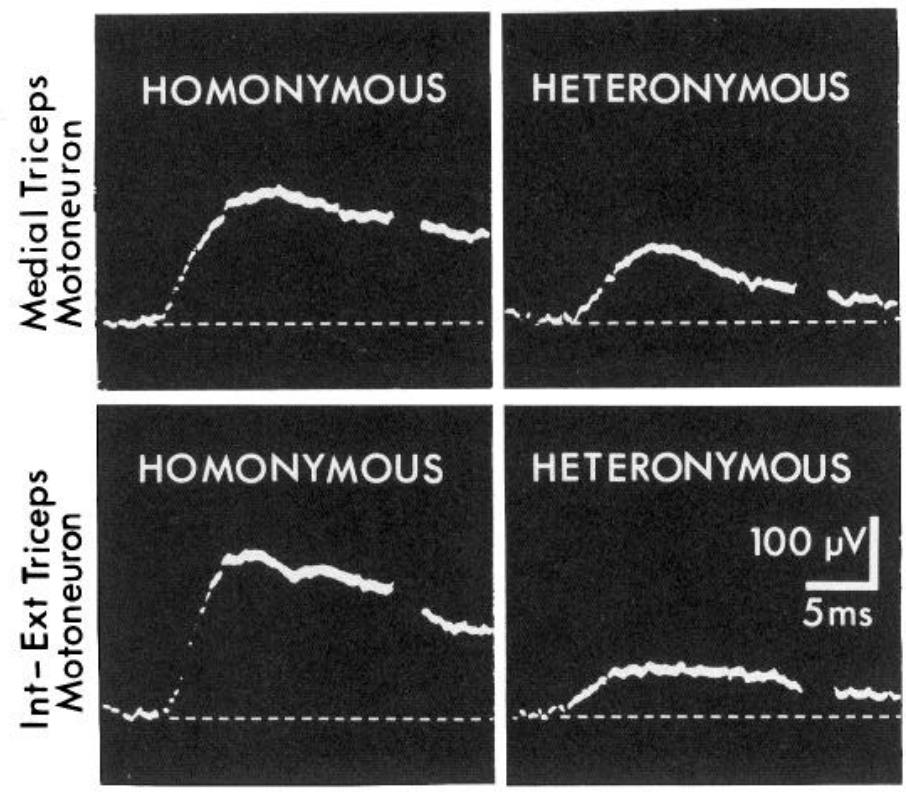

Figure 5. Average EPSPs elicited by stretching homonymous and heteronymous triceps muscles. Many stretch-sensitive axons were activated asynchronously by stretching the entire medial or internal-external muscle. Impulses in any sensory axon from the stretched muscle triggered the signal averager so that these averaged traces represent the input received from an idealized "average" sensory axon from that muscle. Impulse frequencies were kept sufficiently low (by applying only gentle stretches) that the EPSPs did not summate with each other. Homonymous inputs (left side of figure) were excited by stretching the medial head for the medial motoneuron and the combined internal and external heads for the internal-external motoneuron. Similarly, heteronymous inputs (right side of figure) were activated by stretching the other heads. In all cases, the average homonymous synaptic input was of larger amplitude than the average heteronymous one. Calibration pulses (not shown) produced the gaps in the records.

trast to the virtual absence of triceps sensory projections to nearby but unrelated motoneurons.

\section{Numbers of sensory axons in each muscle}

The synaptic efficacy of a group of sensory axons from a particular muscle is related not only to the number of motoneurons each axon innervates and the amplitude of each of these unitary EPSPs but also to the total number of sensory axons in that group. Several lines of evidence suggested that there were substantially more sensory axons innervating the medial triceps muscle as compared to the other two heads of triceps. For example, when the three heads were stretched (by flexing the elbow joint with the muscles intact), the frequency of sensory discharge was greatest in the medial nerve branch, next greatest in the external branch, and least in the internal branch (Fig. 6). Similarly, in the experiment illustrated in Figure 3, we found 11 spots on the medial head from which we could evoke a sensory impulse (Fig. $3 B$ ), whereas only four such spots were found on the combined internal and external heads (Fig. $3 A$ ). We assessed the number of stretch-sensitive sensory axons in each of the triceps heads more directly by several anatomical and physiological techniques.
Muscle spindles. The number of muscle spindles was determined in cross-sections of four triceps muscles. There was a consistent difference in the number of spindles in the three heads. The medial head had $12.4 \pm$ 0.9 intrafusal fiber bundles, the external head had $3.4 \pm$ 0.9 bundles, and the internal head had $1.4 \pm 0.4$ fiber bundles. Each fiber bundle usually has several sensory regions arranged in series, and each sensory region is innervated by a single sensory axon (Gray, 1957). If the number of sensory regions in each bundle is the same for the three muscles, we would expect that the medial head would have many more sensory axons than the external head, and the external head more than the internal head.

Sensory axons. The total number of triceps sensory axons can be estimated more directly by counting the number of labeled myelinated axons in the second dorsal root after the entire triceps nerve is backfilled with horseradish peroxidase. Approximately 30 to 40 axons were labeled in this way (Frank and Westerfield, 1982). To count the number of myelinated sensory axons in
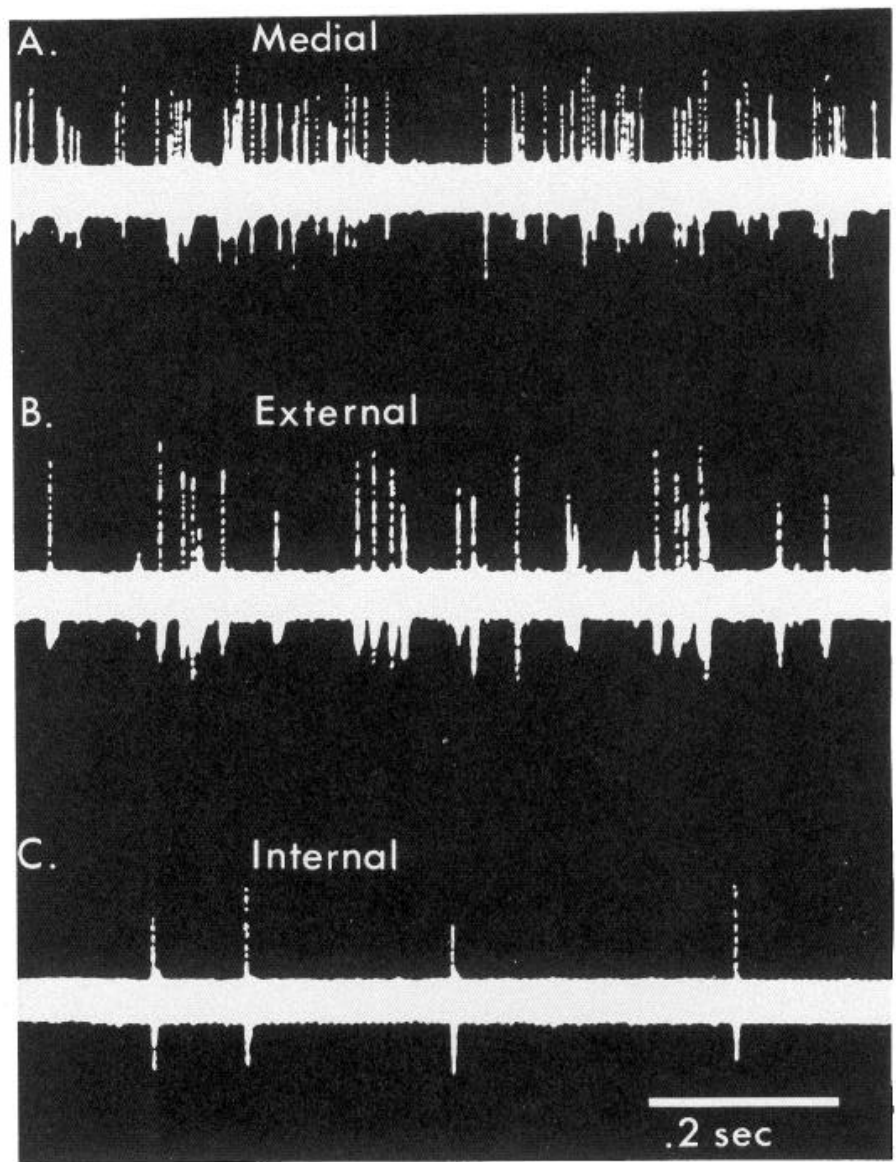

Figure 6. Simultaneous recordings from the nerves supplying the three heads of the triceps muscle while the muscles were stretched by flexing the elbow. The origins and insertions of each muscle were left intact in the arm. There was more evoked impulse activity from the medial head than from the external head, and more from external than from internal. This was presumably the result of a different number of spindles in the three heads. The many different spike heights in the medial nerve recording suggest the activity of many different axons rather than a high rate of activity in a single axon. 
each of the three triceps nerves we first destroyed the motor axons by cutting the ventral root and waiting for them to degenerate (see "Materials and Methods" and Fig. 7). The numbers of myelinated axons remaining in the three triceps nerves of two frogs were 28 and 36 in the medial, 11 and 15 in the external, and 0 and 2 in the internal triceps nerve.

A physiological estimate of the number of sensory axons was made by counting the number of steps in the compound action potential recorded in the cut dorsal root during a gradual increase in the amplitude of the stimulus applied to each triceps nerve. We found $19 \pm 8$ increments when stimulating the medial branch, $10 \pm 2$
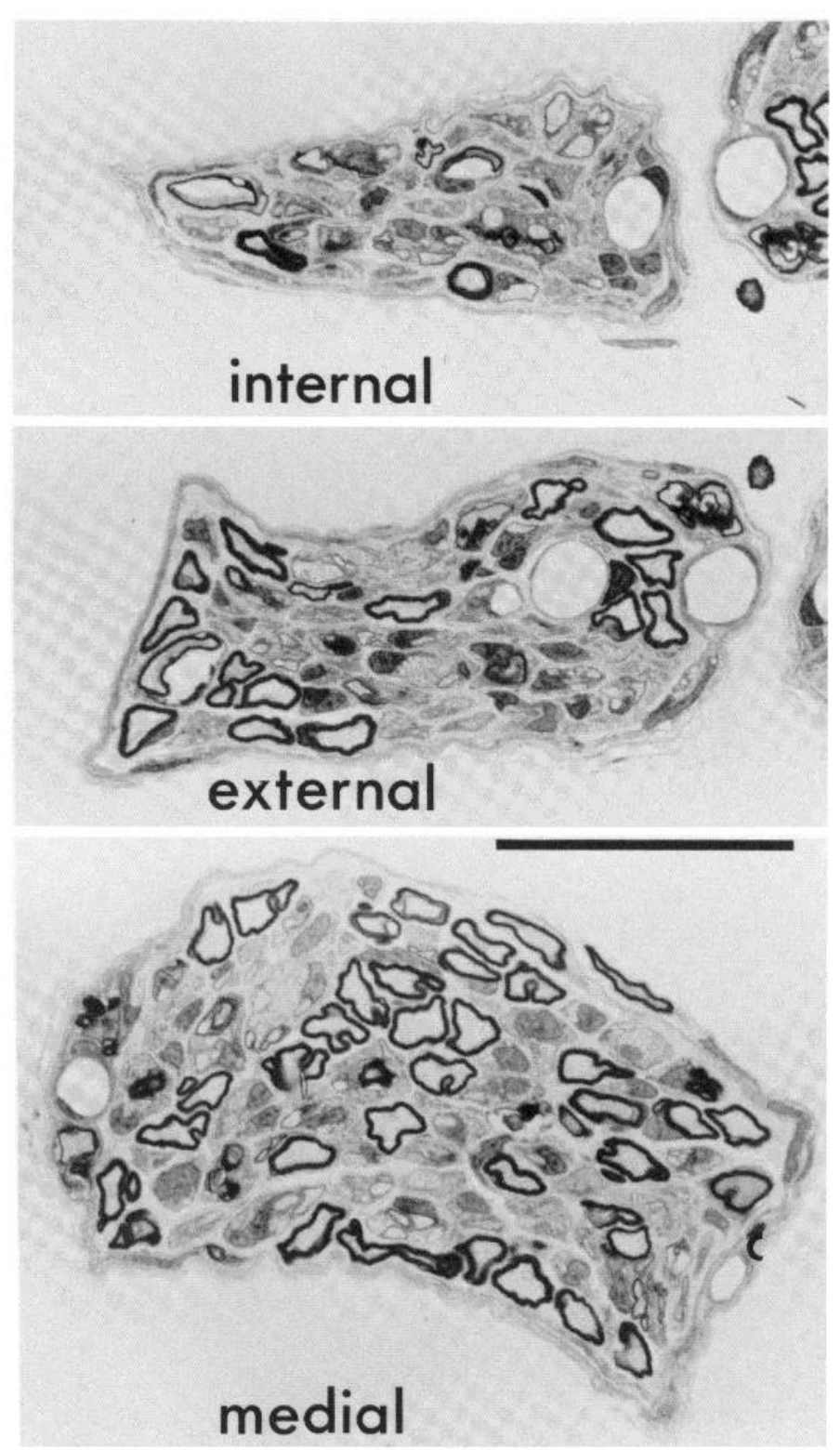

Figure 7. Cross-sections of the nerves supplying the three heads of the triceps muscle. The ventral roots had been cut 2 weeks before the animal was prepared for histology so that remaining profiles are of sensory axons only. There are considerably more myelinated sensory axons projecting to the medial head than to the external head, and more to the external head than to the internal one. Calibration, $50 \mu \mathrm{m}$. for the external branch, and $4 \pm 2$ for the internal branch. This method is likely to underestimate the number of axons (Lichtman, 1977), especially for more than about 10 increments, but does give a relative measure of the numbers of axons in the different branches.

The consistent finding of all of these tests was that the medial head had the most axons and the internal head had the least. The importance of this result is that the relative effects of individual homonymous and heteronymous axons are not directly comparable to the effects of stimulating whole nerves because of a difference in the number of sensory axons in different nerves.

\section{Discussion}

Individual stretch-sensitive afferents from the triceps muscle form highly specific connections with motoneurons in the spinal cord. We have found that afferents from a particular head of triceps preferentially innervate motoneurons projecting to the same head. This preference is evidenced by a greater (nearly complete) projection; each medial triceps afferent projects to almost $95 \%$ of the homonymous motoneurons. Moreover, the synapses formed between afferents and motoneurons tend to be more effective for homonymous than for heteronymous sensory-motor pairs. This connectivity is in striking contrast to the virtual absence of connections between triceps sensory axons and nearby motoneurons which innervate unrelated muscles (see also Frank and Westerfield, 1982).

Our results are similar to those reported for the triceps surae sensory-motor system in the cat. In both systems, muscle sensory fibers project more often to homonymous than to heteronymous motoneurons ( 80 to $95 \%$ projection frequency for homonymous, 50 to $60 \%$ for heteronymous). The amplitudes of nonzero homonymous EPSPs (i.e., excluding the projection failures) in the frog are 80 $\mu \mathrm{V}$ (or about $60 \%$ ) larger, on average, than heteronymous ones. Similarly, in the cat, Scott and Mendell (1976) found that homonymous EPSPs were, on average, $40 \%$ larger than heteronymous ones when measured in the same motoneuron. A more recent study by Munson and Sypert (1979) revealed no significant difference in homonymous versus heteronymous EPSPs, but in this case the potentials were measured in motoneurons innervating different muscles, and the results, therefore, are not strictly comparable.

This difference in homonymous versus heteronymous projections was not related in an obvious way to the location of the synaptic inputs. The relative position of synapses on a motoneuron's dendritic tree can be estimated by measuring the rise times and half-widths of the unitary synaptic potentials (Rall, 1967). Homonymous and heteronymous synaptic potentials in the same motoneuron had similar rise times and half-widths, suggesting that the two types of imputs were located at similar electronic distances from the soma. For example, in the traces shown in Figure 3, the heteronymous inputs had shorter rise times and half-widths than did homonymous ones (rise-times of $6.6 \mathrm{msec}$ versus $4.5 \mathrm{msec}$ halfwidths of $23 \mathrm{msec}$ versus $19 \mathrm{msec}$ ), just opposite to the result required to make homonymous inputs larger. Mendell and co-workers (Scott and Mendell, 1976; Nelson 
and Mendell, 1978), studying the triceps surae system in the cat, have also reported that the location of homonymous versus heteronymous inputs cannot account for the difference in synaptic potential amplitude.

It is interesting to compare the EPSP that would result from simultaneous activation of all the stretch-sensitive axons in a particular nerve branch with that recorded after electrical stimulation of the entire nerve branch. The average unitary heteronymous (medial to internalexternal) EPSP was $78 \mu \mathrm{V}$. With an estimate of 20 to 30 medial stretch-sensitive axons, one would predict a 1.6 to $2.3 \mathrm{mV}$ EPSP if all were activated simultaneously (assuming linear summation). The average input measured after the medial nerve was stimulated electrically was $1.6 \mathrm{mV}$ (Frank and Westerfield, 1982). Similarly, the other heteronymous (internal-external to medial) triceps EPSP would be $55 \mu \mathrm{V} \times 15$ axons, or $0.83 \mathrm{mV}$, compared with $0.86 \mathrm{mV}$ measured directly. Our predictions for the homonymous EPSPs, which could not be measured directly in the earlier study, would be 3.9 to $5.8 \mathrm{mV}$ for the medial and $2.1 \mathrm{mV}$ for the internal-external EPSP. These results suggest that in the frog, as in the cat, most of the monosynaptic input that motoneurons receive from axons in muscle nerves comes from stretch-sensitive axons. Moreover, the contributions from individual axons appear to add approximately linearly with each other.

The number of sensory axons from each of the three heads of the triceps muscle differs considerably. As a result, the specificity shown by sensory axons for particular motoneurons is more obvious when comparing the projections of a single axon to several motoneuronal pools than when comparing the input to one type of motoneuron from several different groups of sensory axons. For example, even though the homonymous projections of internal-external triceps sensory axons are stronger than the heteronymous ones from medial triceps, the total contribution of homonymous axons is no larger than the total heteronymous input. This is because, in this particular case, there are many more individual heteronymous inputs.

A novel feature of these experiments is that we could resolve the smallest averaged synaptic potential evoked by a single sensory axon in a motoneuron. In the cat, published reports suggest that this is not the case. The amplitude histograms in Figure 2 of Scott and Mendell (1976) show many entries in the smallest bin $(0$ to 50 $\mu \mathrm{V})$. Watt et al. (1976) reported that many Ia-motoneuron EPSPs were less than $17 \mu \mathrm{V}$. More recently, Munson and Sypert (1979) reported averaged homonymous and heteronymous EPSPs as small as 5 to $6 \mu \mathrm{V}$. One possible explanation for this result is that frog motoneurons have a higher input impedance than do those in the cat (Coombs et al., 1955; Araki, 1960); the same amount of synaptic current would result in a greater depolarization of the cell.

It is important to point out that these smallest averaged potentials do not correspond directly to a single quantum response, or "unit epsp" described by Kuno (1964) and Jack et al. (1981) in the cat spinal cord and by Shapovalov and Shiriaev (1980) in the frog. The average response will be equal to the unit epsp amplitude times the average number of quanta released (usually denoted by $m$ ). If the probability of release is low, the average response can be considerably smaller than the unit epsp. Our finding of a minimal average response (30 to $40 \mu \mathrm{V}$ ) argues indirectly that the probability of release cannot be arbitrarily small. For example, if a minimal synaptic contact is one synaptic bouton (hence $n=1$ ), and the unit epsp is about $70 \mu \mathrm{V}$ (Shapovalov and Shiriaev, 1980), the the minimal probability of release would be about 0.5 .

At first it might seem surprising that a muscle sensory fiber could manage to innervate virtually all homonymous motoneurons yet form such a relatively weak contact with each onc. In the peripheral nervous system, for example, synaptic connections between pairs of neurons are usually secured with tens or even hundreds of synaptic boutons, and EPSP amplitudes are often an order of magnitude higher than those reported here (see Lichtman and Frank, 1984). However, our results can be explained in a straightforward manner if one assumes that the strength of homonymous versus heteronymous connections is the result simply of the number of synaptic contacts between a sensory and motor neuron. If the average medial triceps homonymous input $(200 \mu \mathrm{V})$ is composed of synaptic contacts each producing an EPSP of 40 to $50 \mu \mathrm{V}$, the connection would be mediated by only four or five such contacts. The Poisson theorem would then predict a projection frequency of 98 to $99 \%$, even greater than the $94 \%$ we observed. Although such an analysis cannot be applied rigorously (because we have no evidence that all individual synaptic contacts produce a 40 to $50 \mu \mathrm{V}$ EPSP, only that the smallest ones do), it suggests that no special mechanism need be invoked to explain our results. A similar analysis has been suggested by Mendell and co-workers (Scott and Mendell, 1976; Nelson and Mendell, 1978) to explain their observations in the cat's spinal cord. $\Lambda$ natomical evidence that this scheme may be correct has been provided by Brown and Fyffe (1981) and Burke and co-workers (Burke et al., 1980; Glenn et al., 1982).

The minimal synaptic contacts determined electrophysiologically should have an anatomical correlate. The minimal contact could be a single synaptic bouton. If a single bouton produced an average response of $40 \mu \mathrm{V}$ (the smallest potentials we recorded), the average synaptic contact between a homonymous medial triceps sensory-motor pair would be mediated by only four or five boutons. By injecting dye into individual triceps sensory and motor neurons we have determined whether such an estimate is in the right range (see Lichtman et al., 1984).

\section{References}

Araki, T. (1960) Effect of electrotonus on the electrical activities of spinal motoneurons of the toad. Jpn. J. Physiol. 10: 518-532.

Brown, A. G., and R. E. W. Fyffe (1981) Direct observations on the contacts made between Ia afferent fibres and $\alpha$ motoneurons in the cat's lumbrosacral spinal cord. J. Physiol. (Lond.) 313: 121-140.

Burke, R. E., M. J. Pinter, A. Lev-Tov, and M. J. O'Donovan (1980) Anatomy of monosynaptic contacts from group Ia afferents to defined types of extensor $\alpha$-motoneurons in the cat. Soc. Neurosci. Abstr. 6: 713. 
Coombs, J. S., D. R. Curtis, and J. C. Eccles (1955) The electrical properties of the motoneurone membrane. J. Physiol. (Lond.) 130: 291-325.

Eccles, J. C., R. M. Eccles, and A. Lundberg (1957) The convergence of monosynaptic excitatory afferents onto many different species of alpha motoneurons. J. Physiol. (Lond.) 137: $22-50$.

Frank, E., and M. Westerfield (1982) Synaptic organization of sensory and motor neurones innervating triceps brachii muscles in the bullfrog. J. Physiol. (Lond.) 324: 479-494.

Glenn, L. L., R. E. Burke, J. W. Fleshman, and A. Lev-Tov (1982) Estimates of electrotonic distance of group Ia contacts on cat $\alpha$-motoneurons: An HRP morphological study. Soc. Nejurosci. Ahstr. 8: 995.

Gray, E. G. (1957) The spindle and extrafusal innervation of a frog muscle. Proc. R. Soc. Lond. (Biol.) 146: 416-430.

Herrera, A. A., and A. D. Grinnell (1980) Differences in synaptic effectiveness at frog neuromuscular junctions: Evidence for long-term physiological regulation. Brain Res. 194: 228231.

Jack, J. J., S. J. Redman, and K. Wong (1981) The components of synaptic potentials evoked in cat spinal motoneurons by impulses in single group Ia afferents. J. Physiol. (Lond.) 321 : 65-96.

Kuno, M. (1964) Quantal components of excitatory synaptic potentials in spinal motoneurones. J. Physiol. (Lond.) 175. 81-99.

Lichtman, J. W. (1977) The reorganization of synaptic connections in the rat submandibular ganglion during post-natal development. J. Physiol. (Lond.) 273: 155-177.

Lichtman, J. W., and E. Frank (1984) On the specificity of sensory-motor and peripheral synaptic connections. In Neuronal Growth and Plasticity, M. Kuno, ed., pp. 149-164, Japan Scientific Societies Press, Tokyo.

Lichtman, J. W., S. Jhaveri, and E. Frank (1984) Anatomical basis of specific connections between sensory axons and motor neurons in the brachial spinal cord of the bullfrog. $J$. Neurosci. 4: 1754-1763.
Lloyd, D. P. C. (1943) Neuron patterns controlling transmission of ipsilateral hind limb.reflexes in cats. J. Neurophysiol. 6: $293-315$.

Lloyd, D. P. C. (1946) Integrative pattern of excitation and inhibition in two-neuron reflex arcs. J. Neurophysiol. 9: 439444.

Mendell, L. M., and E. Henneman (1971) Terminals of single Ia fibers: Location, density and distribution within a pool of 300 homogeneous motoneurons. J. Neurophysiol. 34: 171187.

Munson, J. B., and G. W. Sypert (1979) Properties of single central Ia afferent fibres to motoneurons. J. Physiol. (Lond.) 296: $315-327$.

Nelson, S. G., and L. M. Mendell (1978) Projection of single knee flexor Ia fibers to homonymous and heteronymous motoneurons. J. Neurophysiol. 41: 778-787.

Rall, W. (1967) Distinguishing theoretical synaptic potentials computed for different soma-dendritic distributions of synaptic input. J. Neurophysiol. 30: 1138-1168.

Ridge, R. M. A. P. (1971) Different types of extrafusal muscle fibres in snake costocutaneous muscles. J. Physiol. (Lond.) 217: 393-418.

Scott, J. G., and L. M. Mendell (1976) Individual EPSP's produced by single triceps surae Ia afferent fibers in homonymous and heteronymous motoneurons. J. Neurophysiol. 39: 679-692.

Shapovalov, A. I., and B. I. Shiriaev (1980) Dual mode of junctional transmission at synapses between single primary afferent fibres and motoneurons in the amphibian. J. Physiol. (Lond.) 306: 1-15.

Watt, D. G. D., E. K. Stauffer, A. Taylor, R. M. Reinking, and D. G. Stuart (1976) Analysis of muscle receptor connections by spike-triggered averaging. I. Spindle primary and tendon origin afferents. J. Neurophysiol. 39: 1375-1392.

Westerfield, M., and E. Frank (1982) Specificity of electrical coupling among neurons innervating forelimb muscles of the adult bullfrog. J. Neurophysiol. 48: 904-913. 